\title{
Modeling the Price of Natural Gas with Temperature and Oil Price as Exogenous Factors
}

\author{
Jan Müller, Guido Hirsch and Alfred Müller
}

\begin{abstract}
The literature on stochastic models for the spot market of gas is dominated by purely stochastic approaches. In contrast to these models, Stoll and Wiebauer [14] propose a fundamental model with temperature as an exogenous factor. A model containing only deterministic, temperature-dependent and purely stochastic components, however, still seems not able to capture economic influences on the price. In order to improve the model of Stoll and Wiebauer [14], we include the oil price as another exogenous factor. There are at least two fundamental reasons why this should improve the model. First, the oil price can be considered as a proxy for the general state of the world economy. Furthermore, pricing formulas in oil price indexed gas import contracts in Central Europe are covered by the oil price component. It is shown that the new model can explain price movements of the last few years much better than previous models. The inclusion of oil price and temperature in the regression of a least squares Monte Carlo method leads to more realistic valuation results for gas storages and swing options.
\end{abstract}

Keywords Gas spot price $\cdot$ Oil price model $\cdot$ Temperature $\cdot$ Gas storage valuation Least squares Monte Carlo $\cdot$ Seasonal time series model

\section{Introduction}

During the last years trading in natural gas has become more important. The traded quantities over-the-counter and on energy exchanges have strongly increased and new products have been developed. For example, swing options increase the flexibility of

J. Müller $(\varangle) \cdot$ A. Müller

Department Mathematik, Universität Siegen, Walter-Flex-Str. 3, 57072 Siegen, Germany

e-mail: jan.mueller@uni-siegen.de

A. Müller

e-mail: mueller@mathematik.uni-siegen.de

G. Hirsch

EnBW Energie Baden-Württemberg AG, Durlacher Allee 93, 76131 Karlsruhe, Germany

e-mail: gu.hirsch@enbw.com

K. Glau et al. (eds.), Innovations in Quantitative Risk Management,

Springer Proceedings in Mathematics \& Statistics 99,

DOI 10.1007/978-3-319-09114-3_7 
suppliers and they are used as an instrument for risk management purposes. Important facilities for the security of supply are gas storages.

These are two examples of complex American-style real options that illustrate the need for reliable pricing methods. Both options rely on nontrivial trading strategies where exercise decisions are taken under uncertainty. Therefore, analytic pricing formulas cannot be expected. The identification of an optimal trading strategy under uncertainty is a typical problem of stochastic dynamic programming, but even then numerical solutions are difficult to obtain due to the curse of dimensionality. Therefore, simulation-based approximation algorithms have been successfully applied in this area. Longstaff and Schwartz [9] introduced the least square Monte Carlo method for the valuation of American options. Meinshausen and Hambly [10] extended the idea to Swing options, and Boogert and de Jong [5] applied it to the valuation of gas storages. Their least squares Monte Carlo algorithm requires a stochastic price model for daily spot prices generating adequate gas price scenarios. We prefer this approach to methods using scenario trees or finite differences as it is independent of the underlying price process.

The financial literature on stochastic gas price models is dominated by purely stochastic approaches. The one- and two-factor models by Schwartz [12] and Schwartz and Smith [13] are general approaches applicable to many commodities, such as oil and gas. The various factors represent short- and long-term influences on the price. An important application of gas price models is the valuation of gas storage facilities. Within this context, Chen and Forsyth [7] and Boogert and de Jong [6] propose gas price models. Chen and Forsyth [7] analyze regime-switching approaches incorporating mean-reverting processes and random walks. The class of factor models is extended by Boogert and de Jong [6]. The three factors in their model represent shortand long-term fluctuations as well as the behavior of the winter-summer spread. In contrast to these models, Stoll and Wiebauer [14] propose a fundamental model with temperature as an exogenous factor. They use the temperature component as an approximation of the filling level of gas storages, which have a remarkable influence on the price.

There is a fundamental difference between the model of Stoll and Wiebauer [14] and the other models mentioned before as far as their stochastic behavior is concerned. Incorporating cumulated heating degree days over a winter as an explanatory variable leads to a seasonal effect in the variance of the prices. In this model the variance of the gas prices increases over the winter depending on the actual weather conditions and has a maximum at the end of winter. This is much more in line with the observations than the behavior of the model of Boogert and de Jong [6] where the variance of the gas price has a minimum at the end of winter as there is no effect of the winter-summer spread used there. Another major difference is the use of exogenous variables that can be observed and thus the optimal exercising decision for American-style options depends on these variables and therefore also the price of these real options will be different.

In this paper we extend the model of Stoll and Wiebauer [14] by introducing another exogenous factor to their model: the oil price. There are at least two reasons why we believe that this is useful. The main reason is that an oil price component can 
be considered as a proxy for the state of the world economy in the future. In contrast to other indicators, such as the gross domestic product (GDP), futures prices for oil are available on a daily basis. Furthermore, the import prices for gas in countries such as Germany are known to be oil price indexed.

Apart from the GDP or oil price there might be more candidates as an explanatory variable in the model. The most natural choice would be the forward gas price. We prefer the oil price as it gives us the chance to valuate gas derivatives that are oil price indexed, as is often the case for gas swing contracts. For the valuation of such swing contracts gas price scenarios are needed as well as corresponding oil price scenarios. This application is hardly possible with explanatory variables other than the oil price.

The rest of the paper is organized as follows. In Sect. 2 we introduce the model by Stoll and Wiebauer [14] including a short description of their model for the temperature component. In Sect. 3 we discuss the need for an oil price component in the model. The choice of the component in our model is explained. Then we fit the model to data in Sect. 4. The new model is used within a least squares Monte Carlo algorithm for valuation of gas storages and swing options in Sect. 5. The exogenous factors are included in the regression to approximate the continuation value. We finish with a short conclusion in Sect. 6.

\section{A Review of the Model by Stoll and Wiebauer (2010)}

Modeling the price of natural gas in Central Europe requires knowledge about the structure of supply and demand. On the supply side there are only a few sources in Central Europe, while most of the natural gas is imported from Norway and Russia. On the demand side there are mainly three classes of gas consumers: Households, industrial companies, and gas fired power plants. While households only use gas for heating purposes at low temperatures, industrial companies use gas as heating and process gas. Households and industrial companies are responsible for the major part of the total gas demand.

These two groups of consumers cause seasonalities in the gas price:

- Weekly seasonality: Many industrial companies need less gas on weekends as their operation is restricted to working days.

- Yearly seasonality: Heating gas is needed mainly in winter at low temperatures.

An adequate gas price model has to incorporate these seasonalities as well as stochastic deviations of these.

Stoll and Wiebauer [14] propose a model meeting these requirements and incorporating another major influence factor: the temperature. To a certain extent the temperature dependency is already covered by the deterministic yearly seasonality. This component describes the direct influence of temperature: The lower the temperature, the higher the price. But the temperature influence is more complex than this. A day with average temperature of $0^{\circ} \mathrm{C}$ at the end of a long cold winter has 
a different impact on the price than a daily average of zero at the end of a "warm" winter. Similarly, a cold day at the end of a winter has a different impact on the price than a cold day at the beginning of the winter.

The different impacts are due to gas storages that are essential to cover the demand in winter. The total demand for gas is higher than the capacities of the gas pipelines from Norway and Russia. Therefore, gas providers use gas storages. These storages are filled during summer (at low prices) and emptied in winter months. At the end of a long and cold winter most gas storages will be rather empty. Therefore, additional cold days will lead to comparatively higher prices than in a normal winter.

The filling level of all gas storages in the market would be the adequate variable to model the gas price. However, these data are not available as they are private information. Therefore, we need a proxy variable for it. As the filling levels of gas storages are strongly related to the demand for gas which in turn depends on the temperature, an adequate variable can be derived from the temperature.

Stoll and Wiebauer [14] use normalized cumulated heating degree days to cover the influence of temperature on the gas price. They define a temperature of $15^{\circ} \mathrm{C}$ as the limit of heating. Any temperature below $15^{\circ} \mathrm{C}$ makes households as well as companies switch on their heating systems. Heating degree days are measured by $H D D_{t}=\max \left(15-T_{t}, 0\right)$, where $T_{t}$ is the average temperature of day $t$. As mentioned above the impact on the price depends on the number of cold days observed so far in the winter. In this context, we refer to winter as 1 October and the 181 following days till end of March. We will write $H D D_{d, w}$ for $H D D_{t}$, if $t$ is day number $d$ of winter $w$. Cumulation of heating degree days over a winter leads to a number indicating how cold the winter has been so far. Then we can define the cumulated heating degree days on the day $d$ in winter $w$ as

$$
C H D D_{d, w}=\sum_{k=1}^{d} H D D_{k, w} \text { for } 1 \leq d \leq 182 .
$$

The impact of cumulated heating degree days on the price depends on the comparison with a normal winter. This information is included in normalized cumulated heating degree days

$$
\Lambda_{d, w}=C H D D_{d, w}-\frac{1}{w-1} \sum_{\ell=1}^{w-1} C H D D_{d, \ell} \text { for } 1 \leq d \leq 182
$$

We use $\Lambda_{t}$ instead of $\Lambda_{d, w}$ for simplicity, if $t$ is a day in a winter. The definition of $\Lambda_{t}$ for a summer day is described by a linear return to zero during summer. This reflects the fact that we use $\Lambda_{t}$ as a proxy variable for filling levels of gas storages. Assuming a constant filling rate during summer we thus get the linear part of normalized cumulated heating degree days (see Fig. 1). Positive values of $\Lambda_{t}$ describe winters colder than the average. $\Lambda_{t}$ is included into the gas price model by a regression approach. As the seasonal components and the normalized cumulated 
Fig. 1 Normalized cumulated heating degree days calculated based on temperature data from Eindhoven, Netherlands, for 2003-2011 (Source: Royal Netherlands Meteorological Institute)

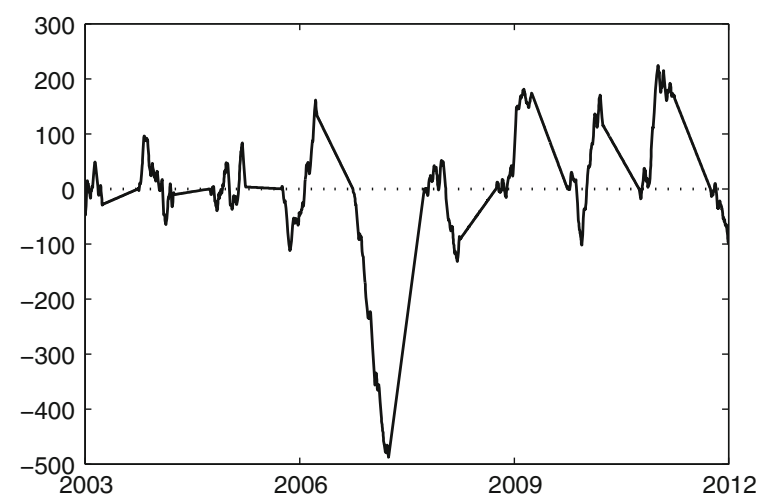

heating degree days are linear with respect to the parameters, we can use ordinary least squares regression for parameter estimation. The complete model can be written as

$$
G_{t}=m_{t}+\alpha \cdot \Lambda_{t}+X_{t}^{(G)}+Y_{t}^{(G)}
$$

with the day-ahead price of gas $G_{t}$, the deterministic seasonality $m_{t}$, the normalized cumulated heating degree days $\Lambda_{t}$, an ARMA process $X_{t}^{(G)}$, and a geometric Brownian motion $Y_{t}^{(G)}$. For model calibration day-ahead gas prices from TTF market (Source: ICE) are used. The Dutch gas trading hub TTF offers the highest trading volumes in Central Europe. As corresponding temperature data we choose daily average temperatures from Eindhoven, Netherlands (Source: Royal Netherlands Meteorological Institute). The fit to historical prices before the crisis can be seen in Fig. 2. Outliers have been removed (see Sect. 4 for details on treatment of outliers).

\section{The Oil Price Dependence of Gas Prices}

The model described in Eq. (3) is capable to cover all influences on the gas price related to changes in temperature. But changes in the economic situation are not covered by that model. This was clearly observable in the economic crisis 2008/2009 (see Fig. 5). During that crisis the demand for gas by industrial companies in Central Europe was falling by more than $10 \%$. As a consequence the gas price rapidly decreased by more than 10 Euro per MWh.

The oil price showed a similar behavior in that period. Economic changes are the main drivers for remarkable changes in the oil price level. Short-term price movements caused by speculators or other effects cause deviations from the price level that represents the state of the world economy. Therefore, gas price changes often correspond to long-term changes in the oil price level. Such an influence can be 


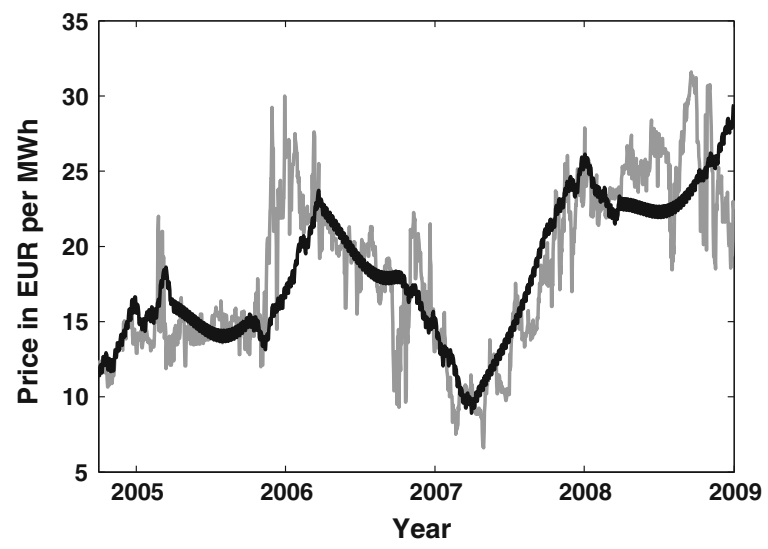

Fig. $2 m_{t}+\alpha \cdot \Lambda_{t}$ from Eq. (3) (black) fitted to TTF prices from 2004-2009 (grey)

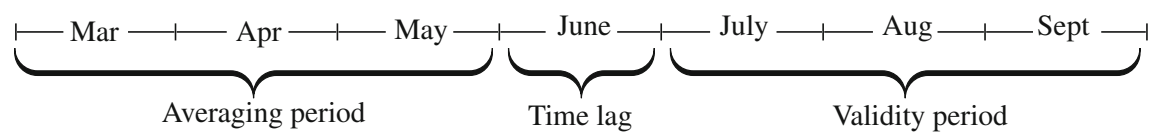

Fig. 3 In a 3-1-3 formula the price is determined by the average price of 3 months (March to May). This price is valid for July-September. The next day of price fixing is 1 October

modeled by means of a moving average of past oil prices. The averaging procedure removes short-term price movements if the averaging period is chosen sufficiently long. The result is a time series containing only the long-term trends of the oil price. Using such an oil price component in a gas price model explains the gas price movements due to changes in the economic situation. This consideration is in line with He et al. [8]. They identify cointegration between crude oil prices and a certain indicator of global economic activity.

Another important argument for the use of this oil price component is based on Central European gas markets. Countries such as Germany import gas via longterm contracts that are oil price indexed. This indexation can be described by three parameters:

1. The number of averaging months. The gas price is the average of past oil prices within a certain number of months.

2. The time lag. Possibly, there is a time lag between the months the average is taken of and the months the price is valid for.

3. The number of validity months. The price is valid for a certain number of months.

An example of a 3-1-3 formula is given in Fig. 3 .

The formulas used in the gas import contracts are not known to all market participants. Theoretically, any choice of three natural numbers is possible. But from other products, like oil price indexed gas swing options, we know that some formulas are more popular than others. Examples of common formulas are 3-1-1, 3-1-3, 6-1-1, 


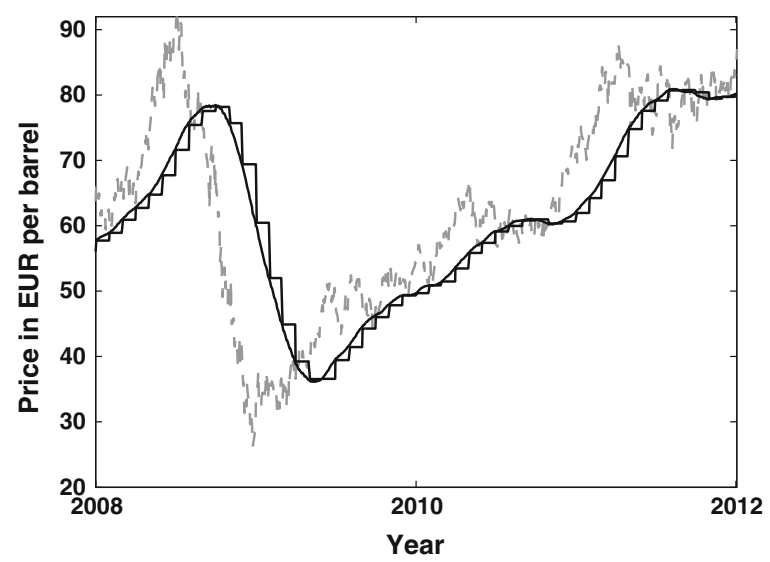

Fig. 4 The oil price (grey), the 6-0-1 formula (black step function) and the moving average of 180 days (black)

6-1-3, and 6-3-3. Therefore, we assume that these formulas are relevant for import contracts as well.

As there are many different import contracts with possibly different price formulas we cannot be sure that one of the mentioned formulas is responsible for the price behavior on the market. The mixture of different formulas might affect the price in the same way as a common formula or a similar one.

Evaluation of the formula leads to price jumps every time the price is fixed. The impact on the gas price will be smoother, however. The new gas price determined on a fixing day is the result of averaging a number of past oil prices. The closer to the fixing day the more prices for the averaging are known. Therefore, market participants have reliable estimations of the new import price. If the new price would be higher it would be cheaper to buy gas in advance and store it. This increases the day-ahead price prior to the fixing day and leads to a smooth transition from the old to the new price level on the day-ahead market.

This behavior of market participants leads to some smoothness of the price. In order to include this fact in a model a smoothed price formula can be used. A sophisticated smoothing approach for forward price curves is introduced by Benth et al. [3]. They assume some smoothness conditions in the knots between different price intervals. It is shown that splines of order four meet all these requirements and make sure that the result is a smooth curve. As our price formulas are step functions like forward price curves, this approach is applicable to our situation.

If the number of validity months is equal to one it is possible to use a moving average instead of a (smoothed) step function to simplify matters (see Fig. 4). This alternative is much less complex than the approach with smoothing by splines, and delivers comparable results. Therefore, the simpler method is applied in case of formulas with one validity month. 
In the next section we compare various formulas regarding their ability to explain the price behavior on the gas market.

\section{Model Calibration with Temperature and Oil Price}

We now compare different formulas of oil prices in the regression model in order to find the one explaining the gas price best (see Fig. 5).

For the choice of the best formula we use the coefficient of determination $R^{2}$ as the measure of goodness-of-fit. We choose the reasonable formula leading to the highest value of $R^{2}$. Reasonable, in this context, means that we restrict our analysis to formulas that are equal or similar to the ones known from other oil price indexed products (compare Sect. 3). The result of this comparison is a 6-0-1 formula (see Fig. 6). Although this is not a common formula there is an explanation for it: The gas price decreased approximately six months later than the oil price in the crisis. This major price movement needs to be covered by the oil price component. As explained above we replace the step function by a moving average. Taking the moving average of 180 days is a good approximation of the 6-0-1 formula. All in all, the oil price component increases the $R^{2}$ as our measure of goodness-of-fit from 0.35 to 0.83 (see Fig. 5). Even if the new model is applied to data before the crisis the oil price component is significant. In that period the increase of $R^{2}$ is smaller but still improves the model.

These comparisons give evidence that both considerations in the previous section are valid. The included oil price component can be seen as the smoothed version of a certain formula. At the same time it can be considered as a variable describing economic influences indicated by the trends and level of the oil price.

Therefore, we model the gas price by the new model

$$
G_{t}=m_{t}+\alpha_{1} \Lambda_{t}+\alpha_{2} \Psi_{t}+X_{t}^{(G)}
$$

with $\Psi_{t}$ being the oil price component. This means that the unobservable factor $Y_{t}^{(G)}$ in Stoll and Wiebauer [14] is replaced by the observable factor $\Psi_{t}$.

Parameter estimation of our model is based on the same data sources as the model by Stoll and Wiebauer [14]. However, we extend the period to 2011. Additionally, we need historical data for the estimation of the oil price component. Therefore, we use prices of the front month contracts of Brent crude oil traded on the Intercontinental Exchange (ICE) from 2002-2011. Using these data we can estimate all parameters applying ordinary least squares regression after removing outliers from the gas price data, $G_{t}$.

Outliers can be due to technical problems or a fire at a major gas storage. We exclude the prices on these occasions by an outlier treatment proposed by Weron [15], where values outside a range around a running median are declared to be outliers. The range is defined as three times the standard deviation. The identified outliers 


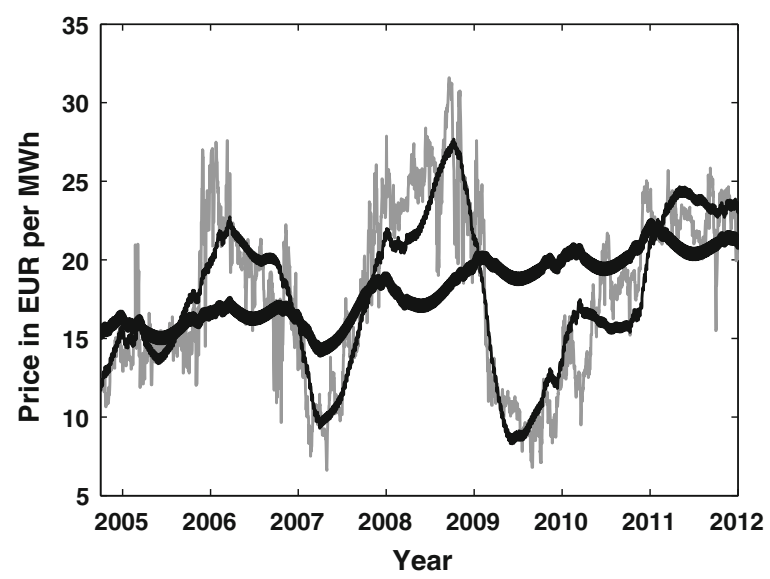

Fig. 5 The model of Stoll and Wiebauer [14] (bold black) and our model (thin black line) fitted to historical gas prices (grey)

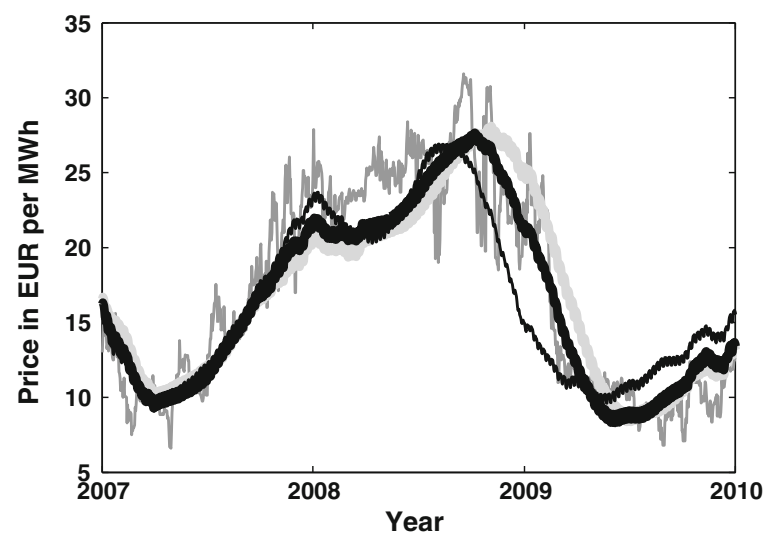

Fig. 6 Comparison of different oil price components in the model: 6-0-1 formula (bold black), 6-1-1 formula (grey) and 3-0-1 formula (thin black) fitted to the historical prices (dark grey)

are excluded in the regression. We do not remove them from our model, however, as they are still included in the estimation of the parameters of the remaining stochastic process.

Altogether, these model components give fundamental explanations for the historical day-ahead price behavior. Short-term deviations are included by a stochastic process (see Sect. 4.3). Long-term uncertainty due to the uncertain development of the oil price is included by the oil price process. Therefore, our model is able to generate reasonable scenarios for the future (see Fig. 7). We specify the stochastic models for the exogenous factors $\Psi_{t}$ and $\Lambda_{t}$ as well as the stochastic process $X_{t}^{(G)}$ in the following. 

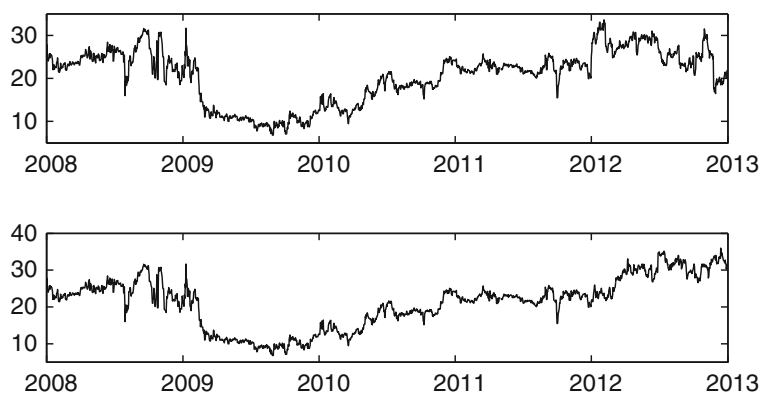

Fig. 7 The historical gas price (2008-2012) and its extensions by two realizations of the gas price process for 2012-2013

\subsection{Oil Price Model}

Oil prices show a different behavior than gas prices, which influences the choice of an adequate model. The most obvious fact is the absence of any seasonalities or deterministic components. Therefore, we model the oil price without a deterministic function or fundamental component. Another major difference affects the stochastic process. While the oil price and also logarithmic oil prices are not stationary the gas price is stationary after removal of seasonalities and fundamental components.

A very common model for nonstationary time series is the Brownian motion with drift applied to logarithmic prices. Drift and volatility of this process can be determined using historical data or by any estimation of the future volatility. For a stationary process, the use of an Ornstein-Uhlenbeck process or its discrete equivalent, an $\mathrm{AR}(1)$ process, is an appropriate simple model.

A combination of these two simple modeling approaches is given by the twofactor model by Schwartz and Smith [13]. They divide the log price into two factors: one for short-term variations and one for long-term dynamics.

$$
\psi_{t}=\exp \left(\chi_{t}+\xi_{t}\right)
$$

with an $\mathrm{AR}(1)$ process $\chi_{t}$ (short-term variations) and a Brownian motion $\xi_{t}$ (longterm dynamics). These processes are correlated. We apply this two-factor model as it considers long- and short-term variations. The estimation of parameters in this model is more complex. The factors are not observable on the market. Following the paper by Schwartz and Smith [13] we apply the Kalman filter for parameter estimation.

The resulting process $\left(\psi_{t}\right)$ is used to derive the process $\left(\Psi_{t}\right)$ in Eq. (4). 

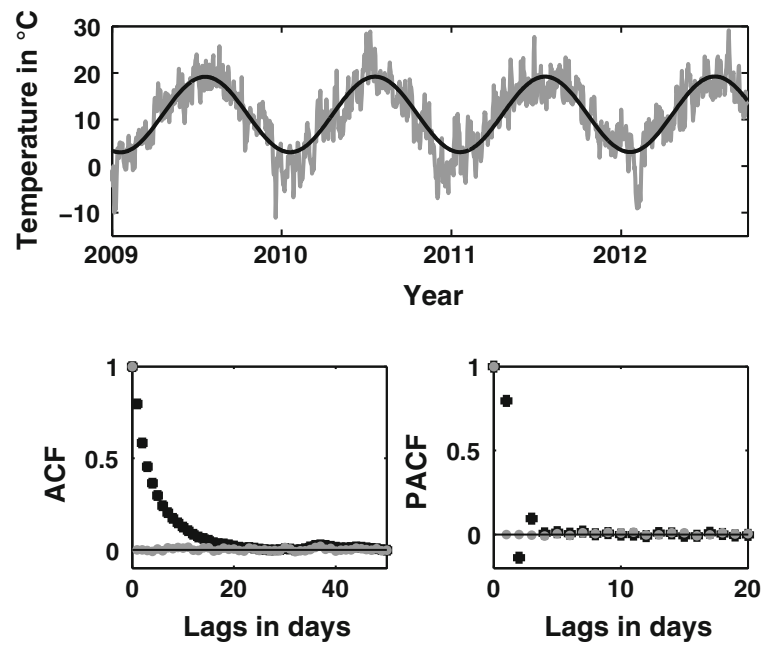

Fig. 8 Top: Fit of deterministic function (black) to the historical daily average temperature ( grey) in Eindhoven, Netherlands. Bottom: Autocorrelation function (left) and partial autocorrelation function (right) of residual time series (black) and innovations of AR(3) process (grey)

\subsection{Temperature Model}

When modeling daily average temperature we can make use of a long history of temperature data. Here, a yearly seasonality and a linear trend can be identified. Therefore, we use a temperature model closely related to the one proposed by Benth and Benth [2].

$$
T_{t}=a_{1}+a_{2} t+a_{3} \sin \left(\frac{2 \pi t}{365.25}\right)+a_{4} \cos \left(\frac{2 \pi t}{365.25}\right)+X_{t}^{(T)}
$$

with $X_{t}^{(T)}$ being an AR(3) process. The model fit with respect to the deterministic part (ordinary least squares regression) and the AR(3) process is shown in Fig. 8. The process $\left(T_{t}\right)$ (see Fig. 9 ) is then used to define the derived process $\left(\Lambda_{t}\right)$ of normalized cumulated heating degree days as described in Sect. 2.

\subsection{The Residual Stochastic Process}

The fit of normalized cumulated heating degree days, oil price component, and deterministic components to the gas price via ordinary least squares regression (see Fig. 10) results in a residual time series. These residuals contain all unexplained, "random" deviations from the usual price behavior. 

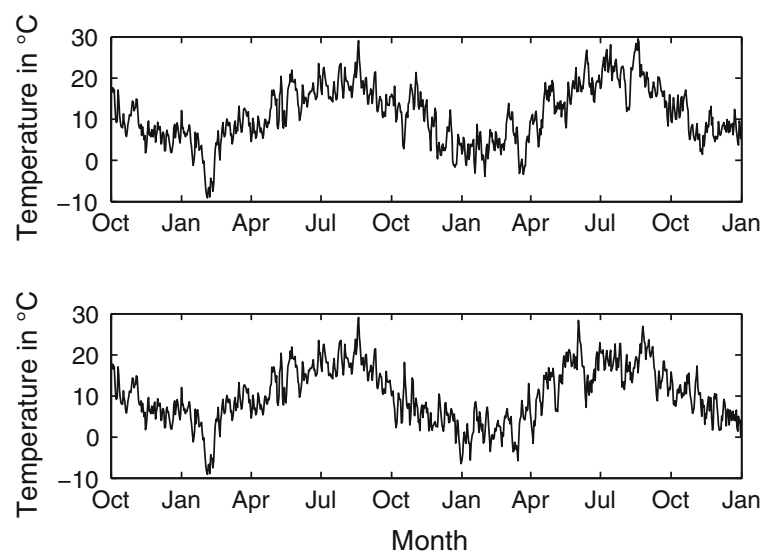

Fig. 9 Historical temperatures and its extension by two realizations of the temperature model

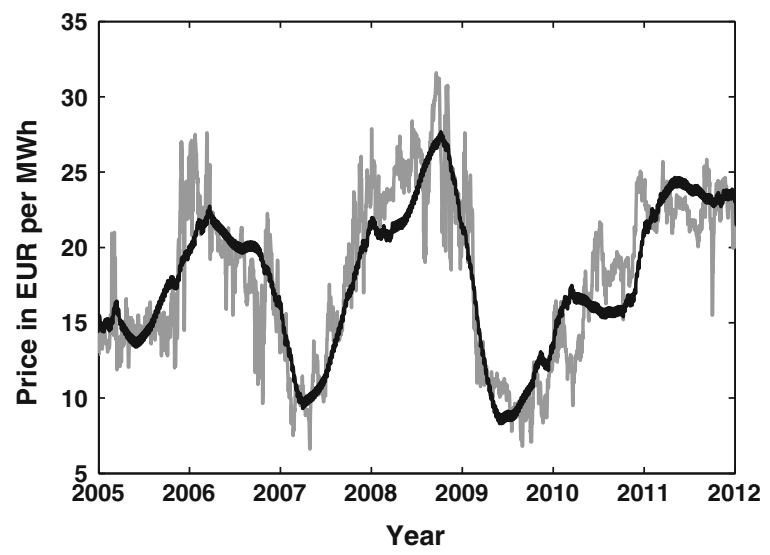

Fig. 10 Fit of deterministic function and exogenous components (black) to the historical gas price (grey)

The residuals exhibit a strong autocorrelation to the first lag. Further analysis of the partial autocorrelation function reveal an $\operatorname{ARMA}(1,2)$ process providing a good fit (see Fig. 11). The empirical innovations of the process show heavier tails than a normal distribution (compare Stoll and Wiebauer [14]). Therefore, we apply a distribution with heavy tails. The class of generalized hyperbolic distributions including the NIG distribution was introduced by Barndorff-Nielsen [1]. The normalinverse Gaussian (NIG) distribution leads to a remarkably good fit (see Fig. 11).

Both the distribution of the innovations and the parameters of autoregressive processes are estimated using maximum likelihood estimation. 

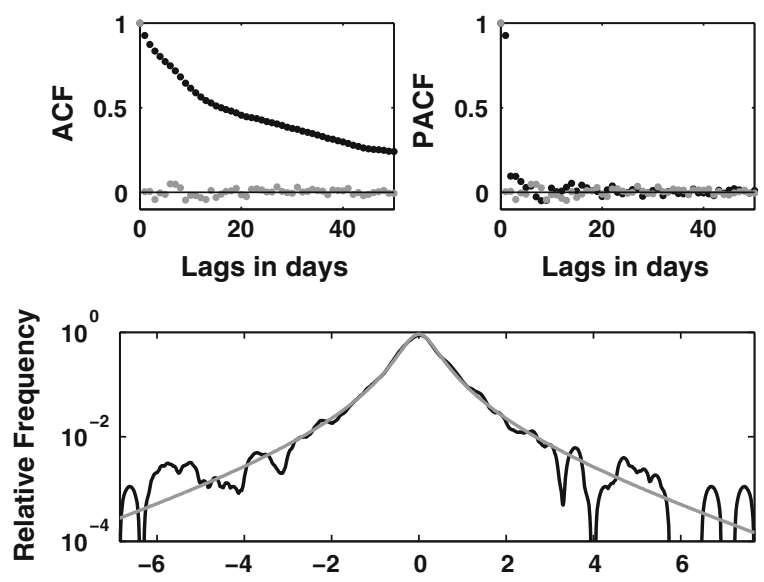

Fig. 11 Top: ACF (left) and PACF (right) of residual time series (black) and innovations of ARMA $(1,2)$ process (grey). Bottom: Fit of NIG distribution (grey) to kernel density of empirical innovations (black)

\section{Option Valuation by Least Squares Monte Carlo Including Exogenous Components}

An optimal exercise of flexibility like gas storages as well as swing options is a decision under uncertainty. While the price for the next day is known, the future development of the spot prices is uncertain. Nevertheless, gas withdrawn today cannot be withdrawn on a day in the future at a possibly higher price level. The identification of an optimal trading strategy under this uncertainty is a typical problem of stochastic dynamic programming, and simulation-based approximation algorithms have been successfully applied in this area. Longstaff and Schwartz [9] introduced the least squares Monte Carlo method for the valuation of American options, Meinshausen and Hambly [10] extended the idea to swing options and Boogert and de Jong [5] applied it to the valuation of gas storages. Furthermore, Boogert and de Jong [6] found that the different components of the gas price process should be included into the regression of the least squares Monte Carlo method for the valuation of gas storages as this increases the value. While they included components that are not observable but virtual components of their price process, the price process introduced in Sect. 4 of this paper includes two exogenous and at the same time observable components. The normalized cumulated heating degree days as well as the 180 days average of the oil price can directly be observed and easily included into the exercise decision of the option that has to be done on a daily basis by a trader. The least squares Monte Carlo method including further factors is described in Sect. 5.1 and valuation examples are given in Sect. 5.2. 


\subsection{Extensions of Least Squares Monte Carlo Algorithm Including Exogenous Components}

A gas storage is characterized by the following restrictions:

- The filling level must lie between given minimum and maximum volumes at any times $0 \leq t \leq T+1: v_{\min }(t) \leq v(t) \leq v_{\max }(t)$

- For each day volume changes are limited by withdrawal and injection rate: $\Delta v_{\min }(t, v(t)) \leq \Delta v \leq \Delta v_{\max }(t, v(t))$

From a mathematical point of view a swing option is a special case of a gas storage. During the delivery period a daily nomination of the gas delivery for the next day is done, while the following restrictions apply:

- Daily contract quantity (DCQ): minimum as well as maximum daily volume; typical values are DCQmin 50-90\% and DCQmax 100-110\% of a given DCQ reference (where DCQ = ACQ/365)

- Annual contract quantity (ACQ): minimum as well as maximum yearly volume; typical values are ACQmin 80-90\% and ACQmax 100-110\% of a given ACQ reference

Due to these restrictions, a swing option is the same as a storage with an initial volume equal to the ACQmax of the swing

$$
v_{\min }(0)=v_{\max }(0)=\mathrm{ACQ} \max
$$

and the following restriction for the final volume

$$
0=v_{\min }(T+1) \leq v(T+1) \leq v_{\max }(T+1)=\mathrm{ACQmin}
$$

where only withdrawal is possible

$$
-\mathrm{DCQ} \max =\Delta v_{\min }(t, v(t)) \leq \Delta v \leq \Delta v_{\max }(t, v(t))=-\mathrm{DCQ} \min
$$

We assume that the storage is available from time $t=0$ till time $t=T+1$ and the holder is allowed to take an action at any discrete date $t=1, \ldots, T$ after the spot price $S(t)$ is known. Let $v(t)$ denote the volume in storage at the start of day $t$ and $\Delta v$ the volume change during day $t$. In case of an injection $\Delta v>0$, while $\Delta v<0$ means withdrawal from the storage. The payoff on day $t$ is

$$
h\left(G_{t}, \Delta v\right)=\left\{\begin{array}{ll}
\left(-G_{t}-c_{W D, t}\right) \cdot \Delta v, & \Delta v \geq 0 \\
\left(-G_{t}-c_{I N, t}\right) \cdot \Delta v, & \Delta v<0
\end{array} .\right.
$$

Here $c_{W D, t}$ denotes the withdrawal costs and $c_{I N, t}$ the injection costs on day $t$, which can be different and may include a bid-ask spread. 
Let $U\left(t, G_{t}, v(t)\right)$ be the value of the flexibility starting at volume level $v(t)$ at time $t$. By $C\left(t, G_{t}, v(t), \Delta v\right)$ we denote the continuation value after taking an allowed action $\Delta v$ from $\mathcal{D}(t, v(t)$ ) (the set of all admissible actions at time $t$ if the filling level is $v(t))$. If $r(t)$ is the interest rate at time $t$ then

$$
C\left(t, G_{t}, v(t), \Delta v\right)=\mathbf{E}\left[e^{-r(t+1)} U\left(t+1, G_{t+1}, v(t)+\Delta v\right)\right] .
$$

The continuation value only depends on $v(t+1):=v(t)+\Delta v$. Therefore, we will from now on also write $C\left(t, G_{t}, v(t+1)\right)$ for short. With this notation the flexibility value $U\left(t, G_{t}, v(t)\right)$ satisfies the following dynamic program:

$$
\begin{aligned}
U\left(T+1, G_{T+1}, v(T+1)\right) & =q\left(G_{T+1}, v(T+1)\right) \\
U\left(t, G_{t}, v(t)\right) & =\max _{\Delta v \in \mathcal{D}(t, v(t))}\left[h\left(G_{t}, \Delta v\right)+C\left(t, G_{t}, v(t), \Delta v\right)\right]
\end{aligned}
$$

for all times $t$. In the first equation $q$ is a possible penalty depending on the volume level at time $T+1$ and the spot price at this time $G_{T+1}$.

As the continuation value cannot be determined analytically, we use the least squares Monte Carlo method to approximate the continuation value

$$
C\left(t, G_{t}, v(t+1)\right) \approx \sum_{l=0}^{m} \beta_{l, t}(v(t+1)) \cdot \phi_{l}\left(G_{t}\right)
$$

using basis functions $\phi_{l}$. If $N$ price scenarios are given, estimates $\hat{\beta}_{l, t}(v(t+1))$ for the coefficients $\beta_{l, t}(v(t+1))$ result by regression. With these coefficients an approximation $\hat{C}\left(t, G_{t}, v(t+1)\right)$ of the continuation value is obtained that is used to determine an approximately optimal action $\Delta v(t)$ for all volumes $v(t)$.

Moreno and Navas [11] have shown that the concrete choice of the basis functions does not have much influence on the results. For this reason we have chosen the easy to handle polynomial basis functions $\phi_{l}\left(G_{t}\right)=G_{t}^{l}$. Calculations have shown that $m=3$ is enough to get good results. A higher number of basis functions leads to similar results.

Boogert and de Jong [6] use a multi-factor price process and include the factors of the price process into the basis used for the regression in the least squares Monte Carlo method. While their factors are unobservable, our price process (see Eq. (4)) includes two exogenous factors, which can easily be observed. We include the oil price component $\Psi_{t}$ (see Sect.3) and the temperature component $\Lambda_{t}$ (see Sect.2) into the regression by using 


$$
\begin{aligned}
C\left(t, G_{t}, \Lambda_{t}, \Psi_{t}, v(t), \Delta v\right) \approx & \sum_{l=0}^{m} \beta_{l, t} \cdot \phi_{l}\left(G_{t}\right) \\
& +\beta_{m+1, t} \Psi_{t}+\beta_{m+2, t} \Psi_{t}^{2}+\beta_{m+3, t} \Psi_{t} \cdot G_{t} \\
& +\beta_{m+4, t} \Lambda_{t}+\beta_{m+5, t} \Lambda_{t} \cdot G_{t} .
\end{aligned}
$$

For simplification of notation we omit to mention the explicit dependence of the parameters on the filling level $v(t+1)$ as is done in Boogert and de Jong [6] throughout the paper. Monomials of higher degree in the oil price or temperature components as well as higher mixed terms have also been examined, but do not yield better results.

\subsection{Influence of Exogenous Components on Valuation Results}

Gas storages and swing options are not only virtual products but are real options. This means that traders need to take exercise decisions on a daily basis. These decisions depend on all observable market information. In order to reflect this behavior in the pricing algorithm for such options we will use the least squares Monte Carlo method described above in combination with the spot price model in Sect. 4. The examples given in this section are artificial gas storages and swing options valuated at two different dates, 4 July 2012 and 2 April 2013. These dates are characterized by a very different implicit volatility observed at the markets-for example for TTF the long-term volatility has significantly decreased in the 8-month period from 25 to $12 \%$ (Source: ICE). At the same time the summer-winter spread between winter 13/14 and summer 13 has decreased from 2.40 EUR/MWh to 1.20 EUR/MWh, whereas the price level has increased from 26.15 EUR/MWh to 27.70 EUR/MWh.

The TTF market prices have been used for the valuation of a slow and a fast storage that are identical to the ones valued by Boogert and de Jong [6]. Moreover, we have also valued a flexible and an inflexible swing contract. The parameters for these storages and swings are given in Table 1. All valuations have been done using 5,000 price scenarios, which results in sufficiently convergent results.

We denote by daily intrinsic the value obtained if a daily price forward curve is taken and an optimal exercise is calculated (using a deterministic dynamic program). This value could be logged in immediately if each single future day could be traded as an individual forward contract. The fair value denotes the value resulting from the least squares Monte Carlo method, and the extrinsic value is the difference between fair value and daily intrinsic value. Therefore, the extrinsic value is a measure for the value of the flexibility included in the considered real option.

As can clearly be seen by comparing Tables 2 and 3 the decrease of the summerwinter spread results in a lower daily intrinsic value for the storages. In contrast to this behavior the intrinsic value of the flexible swing increases because of the higher price level in 2013 compared to 2012. Furthermore, the decrease of volatility does 
Table 1 Parameters for gas storages and swing options from 1.4.2013-1.4.2014

\begin{tabular}{l|l|l|l|l}
\hline Parameter & Slow storage & Fast storage & Inflexible swing & Flexible swing \\
\hline Min volume & $0 \mathrm{MWh}$ & $0 \mathrm{MWh}$ & $0 \mathrm{MWh}$ & $0 \mathrm{MWh}$ \\
\hline Max volume & $100 \mathrm{MWh}$ & $100 \mathrm{MWh}$ & $438 \mathrm{MWh}$ & $438 \mathrm{MWh}$ \\
\hline Min injection & $0 \mathrm{MWh} /$ day & $0 \mathrm{MWh} /$ day & - & - \\
\hline Max injection & $1 \mathrm{MWh} /$ day & $2 \mathrm{MWh} /$ day & - & - \\
\hline Min withdrawal & $0 \mathrm{MWh} /$ day & $0 \mathrm{MWh} /$ day & $0.6 \mathrm{MWh} /$ day & $0 \mathrm{MWh} /$ day \\
\hline Max withdrawal & $1 \mathrm{MWh} /$ day & $5 \mathrm{MWh} /$ day & $1.2 \mathrm{MWh} /$ day & $1.2 \mathrm{MWh} /$ day \\
\hline Injection costs & $0 \mathrm{EUR} / \mathrm{MWh}$ & $0 \mathrm{EUR} / \mathrm{MWh}$ & - & - \\
\hline Withdrawal costs & $0 \mathrm{EUR} / \mathrm{MWh}$ & $0 \mathrm{EUR} / \mathrm{MWh}$ & $27 \mathrm{EUR} / \mathrm{MWh}$ & $27 \mathrm{EUR} / \mathrm{MWh}$ \\
\hline Start volume & $0 \mathrm{MWh}$ & $0 \mathrm{MWh}$ & $438 \mathrm{MWh}$ & $438 \mathrm{MWh}$ \\
\hline Max end volume & $0 \mathrm{MWh}$ & $0 \mathrm{MWh}$ & $146 \mathrm{MWh}$ & $146 \mathrm{MWh}$ \\
\hline
\end{tabular}

Table 2 Results for valuation date 4 July 2012 (5,000 scenarios)

\begin{tabular}{l|l|l|l|c}
\hline Contract & Factors in regression & Daily intrinsic & Fair value & Extrinsic value \\
\hline \multirow{2}{*}{ Slow storage } & Spot & 360.8 & 382.4 & 21.6 \\
\cline { 2 - 5 } & Spot \& Brent & 360.8 & 549.5 & 188.7 \\
\cline { 2 - 5 } & Spot \& Brent \& HDD & 360.8 & 571.2 & 210.4 \\
\hline Fast storage & Spot & 517.1 & 561.8 & 44.7 \\
\cline { 2 - 5 } & Spot \& Brent & 517.1 & $1,006.6$ & 489.5 \\
\cline { 2 - 5 } & Spot \& Brent \& HDD & 517.1 & $1,090.1$ & 572.9 \\
\hline \multirow{2}{*}{ Flexilexible swing } & Spot & -126.2 & 274.5 & 400.7 \\
\cline { 2 - 5 } & Spot \& Brent & -126.2 & 285.4 & 411.6 \\
\cline { 2 - 5 } & Spot \& Brent \& HDD & -126.2 & 286.3 & 412.4 \\
\hline & Spot & -41.6 & 356.5 & 398.1 \\
\cline { 2 - 5 } & Spot \&Brent & -41.6 & 397.2 & 438.8 \\
\cline { 2 - 5 } & Spot \&Brent \&HDD & -41.6 & 959.6 & $1,001.2$ \\
\hline
\end{tabular}

not change the extrinsic value of the two storages - very much in contrast to the swings.

For storages these findings correspond very well to the observations by Boogert and de Jong [6]. They also found that a change of volatility in the long-term component does not influence the value of gas storages - it may even decrease the value. An explanation for this behavior is that it becomes more difficult for traders to decide correctly if today's price is high or low and therefore withdrawal, injection, or no action makes most sense. Due to the decision under uncertainty about the future price development with an increased volatility, more and more wrong decisions are taken and this may decrease the value at least in case of fast storages.

The situation is completely different for swing options. With an increasing volatility their value also increases. This is not surprising as can easily be seen from looking at a special case. If the yearly restriction is not binding the swing is equivalent to a 
Table 3 Results for valuation date 2, April 2013 (5,000 scenarios)

\begin{tabular}{l|l|l|l|l}
\hline Contract & Factors in regression & Daily intrinsic & Fair value & Extrinsic value \\
\hline \multirow{2}{*}{ Slow storage } & Spot & 227.3 & 309.5 & 82.2 \\
\cline { 2 - 5 } & Spot \& Brent & 227.3 & 419.1 & 191.8 \\
\cline { 2 - 5 } & Spot \& Brent \& HDD & 227.3 & 411.7 & 184.4 \\
\hline \multirow{2}{*}{ Fast storage } & Spot & 353.5 & 593.4 & 240.0 \\
\cline { 2 - 5 } & Spot \& Brent & 353.5 & 855.0 & 501.6 \\
\cline { 2 - 5 } & Spot \& Brent \& HDD & 353.5 & 877.0 & 523.5 \\
\hline \multirow{2}{*}{ Flexible swing } & Spot & 310.0 & 485.2 & 175.2 \\
\cline { 2 - 5 } & Spot \& Brent & 310.0 & 488.0 & 177.9 \\
\cline { 2 - 5 } & Spot \& Brent \& HDD & 310.0 & 471.9 & 161.9 \\
\cline { 2 - 5 } & Spot & 324.1 & 542.1 & 218.0 \\
\cline { 2 - 5 } & Spot \& Brent & 324.1 & 558.5 & 234.4 \\
\cline { 2 - 5 } & Spot \& Brent \& HDD & 324.1 & 572.2 & 248.1 \\
\hline
\end{tabular}

strip of European options. In this case it is well known that an increase of volatility implies an increase of the extrinsic option value under quite general assumptions on the underlying stochastic process, see e.g. Bergenthum and Rüschendorf [4].

Another important difference between swings and storages is their behavior if the exogenous components of the spot price process are included in the regression of the algorithm. For the value of storages the oil price component is much more important-in contrast to swings. For the inflexible swing both components are irrelevant, while for the flexible swing the temperature component is more important than the oil price component. For storages the oil price component is a measure for normal long-term levels. As prices revert back to this long-term level mainly defined by the oil price component, a price higher than this level is good for withdrawal while a price lower than this level is good for injection. Therefore, an inclusion in the regression is very important for the exercise decision and increases the value.

Another interesting observation is the influence of the two exogenous components on the less flexible products. While an inclusion of the oil price component increases the fair value, a further inclusion of the temperature component decreases the value slightly for valuation date 2 April 2013 - but not for 4 July 2012. One important reason is that in April 2013 the end of a long and as far as heating degrees are concerned quite normal winter has just been exceeded and the linear return to zero is starting, while the winter 2011/12 has been very warm and in July the linear return with a slight gradient has half been finished.

To sum up, these results indicate that it is very important to include both exogenous components into the exercise decision for storages as well as swings, as this can significantly increase the extrinsic value. 


\section{Conclusion}

The spot price model by Stoll and Wiebauer [14] with only temperature as an exogenous factor is not able to explain the gas price behavior during the last years. We have shown that adding an oil price component as another exogenous factor remarkably improves the model fit. It is not only a good proxy for economic influences on the price but also approximates the oil price indexation in gas import contracts on Central European gas markets. These fundamental reasons and the improvement of model fit give justification for the inclusion of the model component. The resulting simulation paths from the model are reliable. The inclusion of both exogenous factors in algorithms for the valuation of options by least squares Monte Carlo remarkably affects the valuation results.

Open Access This chapter is distributed under the terms of the Creative Commons Attribution Noncommercial License, which permits any noncommercial use, distribution, and reproduction in any medium, provided the original author(s) and source are credited.

\section{References}

1. Barndorff-Nielsen, O.E.: Hyperbolic distributions and distributions on hyperbolae. Scand. J. Stat. 5, 151-157 (1978)

2. Benth, F.E., Benth, J.S.: The volatility of temperature and pricing of weather derivatives. Quant. Financ. 7, 553-561 (2007)

3. Benth, F.E., Koekkebakker, S., Ollmar, F.: Extracting and applying smooth forward curves from average-based commodity contracts with seasonal variation. J. Deriv. 15, 52-66 (2007)

4. Bergenthum, J., Rüschendorf, L.: Comparison of option prices in semimartingale models. Financ. Stochast. 10, 222-249 (2006)

5. Boogert, A., de Jong, C.: Gas storage valuation using a Monte Carlo method. J. Deriv. 15, 81-98 (2008)

6. Boogert, A., de Jong, C.: Gas storage valuation using a multifactor price process. J. Energy Mark. 4, 29-52 (2011)

7. Chen, Z., Forsyth, P.A.: Implications of a regime-switching model on natural gas storage valuation and optimal operation. Quant. Financ. 10, 159-176 (2010)

8. He, Y., Wang, S., Lai, K.K.: Global economic activity and crude oil prices: a cointegration analysis. Energy Econ. 32, 868-876 (2010)

9. Longstaff, F.A., Schwartz, E.S.: Valuing American options by simulation: a simple least-squares approach. Rev. Financ. Stud. 14, 113-147 (2001)

10. Meinshausen, N., Hambly, B.M.: Monte Carlo methods for the valuation of multiple-exercise options. Math. Financ. 14, 557-583 (2004)

11. Moreno, M., Navas, J.F.: On the robustness of least-squares Monte Carlo for pricing American derivatives. Rev. Deriv. Res. 6, 107-128 (2003)

12. Schwartz, E.S.: The stochastic behaviour of commodity prices: implications for valuation and hedging. J. Financ. 52, 923-973 (1997) 
13. Schwartz, E.S., Smith, J.E.: Short-term variations and long-term dynamics in commodity prices. Manage. Sci. 46, 893-911 (2000)

14. Stoll, S.O., Wiebauer, K.: A spot price model for natural gas considering temperature as an exogenous factor and applications. J. Energy Mark. 3, 113-128 (2010)

15. Weron, R.: Modeling and Forecasting Electricity Loads and Prices: A Statistical Approach. Wiley, Chichester (2006) 